\title{
Reconstruction of nerve root sheaths for sacral extradural spinal meningeal cysts with spinal nerve root fibers
}

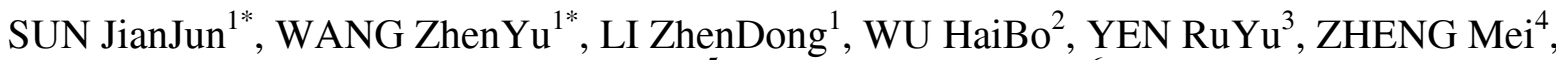 \\ CHANG Qing ${ }^{5} \&$ LIU Isabelle Yisha ${ }^{6}$ \\ ${ }^{1}$ Department of Neurosurgery, Peking University Third Hospital, Peking University, Beijing 100191, China; \\ ${ }^{2}$ Department of Neuroradiology, Peking University Third Hospital, Peking University, Beijing 100191, China; \\ ${ }^{3}$ Department of General Surgery, Peking University Third Hospital, Peking University, Beijing 100191, China \\ ${ }^{4}$ Department of Neurology, Peking University Third Hospital, Peking University, Beijing 100191, China; \\ ${ }^{5}$ Department of Pathology, Peking University Third Hospital, Peking University, Beijing 100191, China; \\ ${ }^{6}$ Department of Head \& Neck Surgery, UCLA Medical Center, Los Angeles, CA 90095, USA
}

Received July 6, 2013; accepted July 18, 2013; published online September 3, 2013

\begin{abstract}
This study analyzed the clinical characteristics and outcomes of sacral extradural spinal meningeal cysts with spinal nerve root fibers treated by reconstruction of the nerve root sheaths. The relationships between the cysts and spinal nerve root fibers were examined microscopically, the cysts were partially excised, and the defects were oversewn to reconstruct the nerve root sheaths. The Improved Japanese Orthopedic Association (IJOA) scoring system was used to evaluate preoperative and postoperative neurological function. Thirty-eight patients were included in this study, with a mean age of $41.4 \pm 15.57$ years. The mean IJOA score was $18.8 \pm 1.32$ preoperatively and $19.6 \pm 0.65$ postoperatively, which was a significant difference $(t=-3.77, P=0.001)$. These results indicate a significant improvement in neurological function after surgery. The most significant improvement in neurological function was sensation $(z=-2.86, P=0.004)$, followed by bowel/bladder function $(z=-2.31, P=0.02)$.
\end{abstract}

sacral canal, spinal meningeal cysts, reconstructed nerve root sheath, spinal nerve root fibers, clinical outcomes

Citation: Sun J J, Wang Z Y, Li Z D, et al. Reconstruction of nerve root sheaths for sacral extradural spinal meningeal cysts with spinal nerve root fibers. Sci China Life Sci, 2013, 56: 1007-1013, doi: 10.1007/s11427-013-4536-7

Sacral extradural spinal meningeal cysts (SESMCs) are extradural meningeal cysts located in the sacral canal. These cysts are commonly incidental findings on magnetic resonance imaging (MRI) examinations, and are usually asymptomatic. According to the classification of sacral spinal canal cysts by Nabors et al. [1], SESMCs are divided into two types: those with spinal nerve root fibers (SNRFs) and those without. SESMCs with SNRFs, also known as Tarlov cysts, are characterized by collection of cerebrospinal fluid (CSF) between the endoneurium and perineurium

*Corresponding author (email: sunjj2008@gmail.com; wzyu502@hotmail.com) of the nerve root sheath near the dorsal root ganglion [2].

The pathophysiological mechanism underlying the formation of SESMCs with SNRFs is congenital weakness of the nerve root sheath [3]. High hydrostatic pressure generated by upright walking and a ball-valve effect result in cyst enlargement [3-5]. The walls of the cysts are formed by enlargement of the perineural nerve root sheaths, resulting in alteration of the environment around the spinal nerve root fibers. This alteration causes malfunction of the involved spinal nerve root fibers, similar to axonal transport dysfunction [6]. This malfunction differs from malfunction associated with compression against adjacent bone or other nerve 
roots. It would be useful to determine methods of restoring the normal environment of spinal nerve root fibers by surgical treatment of SESMCs.

Despite advances in diagnostic techniques, the optimal treatment of symptomatic SESMCs remains unclear. Nonsurgical options include lumbar CSF drainage and computed tomography (CT)-guided percutaneous cyst aspiration with or without infusion of fibrin glue, but these do not prevent recurrence of symptomatic cysts [7]. The goals of surgical intervention of symptomatic SESMCs are to relieve nerve stimulation and compression, and stop bone erosion. Neurosurgical techniques for treating symptomatic SESMCs include lumbar-peritoneal shunt placement, simple decompressive laminectomy, partial cyst removal and neck ligation with or without nerve root resection, cyst wall excision, cyst fenestration, and cyst shrinkage using bipolar cautery [4]. Although there is no consensus regarding the definitive treatment of symptomatic SESMCs, several previous studies have reported that surgical methods have the best long-term outcomes [1-2,7].

Most previous reports describing the management of SESMCs presented either single cases or a small series of no more than 20 cases [2,4,7-11]. In this study, we aimed to identify and treat a larger cohort of patients with SESMCs and prospectively study their clinical outcomes, to evaluate the treatment of this rare disorder.

Regardless of the nomenclature or classification system, the defining feature of Tarlov cysts (SESMCs with SNRFs) is the presence of SNRFs within the cyst wall or cyst cavity. Although CT myelography and MRI are useful diagnostic tools, the final diagnosis of Tarlov cysts (as opposed to SESMCs without SNRFs) can only be made intraoperatively under the surgical microscope [3]. This study aimed to investigate the clinical manifestations, surgical techniques, and outcomes of 38 patients with SESMCs with SNRFs.

\section{Materials and methods}

\subsection{Study design}

This was a prospective cohort study using clinical records. Approval for the study was obtained from the Research Ethics Board of Peking University Third Hospital. All subjects provided written informed consent for surgical operation and medical photography as well as inclusion in the prospective cohort clinical study.

\subsection{Patients}

From June 2009 to October 2012, a consecutive series of 38 patients who were diagnosed with SESMCs with SNRFs underwent nerve root sheath reconstruction. Basic demographic, clinical and radiological data were evaluated. Preoperative neuroimaging including MRI was performed in all patients. The period of symptoms before presentation was defined as the time from the onset of symptoms to the time of presentation, and was recorded in months.

\subsection{Inclusion and exclusion criteria}

Patients were included in the study if they met the following criteria: (i) radiological findings consistent with SESMCs, (ii) neurological symptoms attributable to SESMCs, and (iii) SESMCs with SNRFs observed under the operating microscope. Patients were excluded if their symptoms could not be differentiated from lumbar spinal stenosis or lumbar intervertebral disc herniation. The SESMCs with SNRFs included two subgroups: paraneural and perineural cysts. The clinical data were collected prospectively by clinicians who were blind to the SNRF status of the patients. The data were then analyzed using appropriate statistical methods.

\subsection{Clinical presentation}

The initial presenting symptoms, location of presenting symptoms, and period of symptoms before initial presentation were recorded. The presenting symptoms were categorized into (i) bowel/bladder and sexual dysfunction, (ii) lower extremity weakness, (iii) lower extremity numbness, (iv) pain, (v) bearing-down feeling, and (vi) more than one symptom. The locations of the presenting symptoms were categorized as (i) sacrococcygeal, (ii) perineum and external genitalia, (iii) lower waist, (iv) buttocks, (v) legs, or (vi) more than one region.

Abnormalities on neurological examination were classified as sensory dysfunction, lower extremity weakness, and bowel/bladder dysfunction. The IJOA scoring system was used to evaluate preoperative and postoperative neurological function. The IJOA is based on the JOA scoring system, with the addition of a score for bowel function as normal, slightly dysfunctional, severely dysfunctional, or incontinent [10].

\subsection{Surgical techniques}

Our operative technique follows the standard procedures for SESMC surgery. An incision is made from L5 to S3, and the sacral laminae are completely exposed according to the location of the SESMCs. Laminectomy is performed with a rongeur, while carefully preserving the integrity of the underlying cysts. The surgical microscope is then brought into the field. The terminal thecal sac is identified and dissected free from the overlying cysts. Each cyst is dissected from the surrounding structures to reveal its origin and relationships with the SNRFs (Figure 1) by Dr. Sun JianJun and Dr. Wang Zhen $\mathrm{Yu}$. The cysts are partially resected and the defect is oversewn to prevent leakage of CSF from the subarachnoid space and to reconstruct the nerve root sheath. Redundant cyst wall is shrunk using bipolar cautery. Intraoperative neurophysiological monitoring is used to dif- 


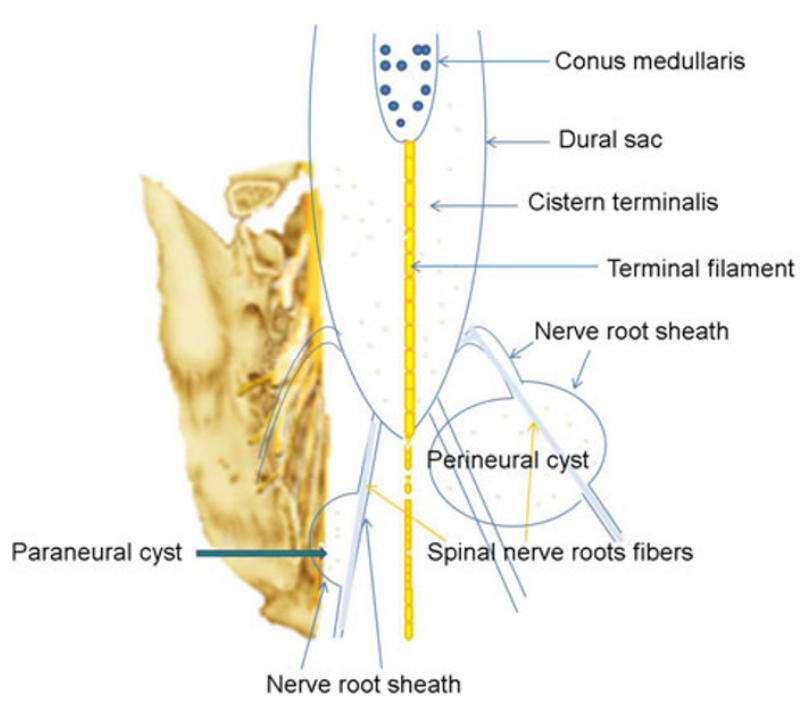

Figure 1 Two types of sacral extradural spinal meningeal cysts with spinal nerve root fibers.

ferentiate SNRFs from other tissues, and electrical stimulation is used to verify that no motor nerve fibers are involved. The closure is reinforced with a local muscle flap.

\subsection{Characteristics of SESMCs}

Under the surgical microscope, the number, maximum diameter, and types of cyst were determined. If there were multiple cysts in the sacral canal, the maximum diameter, type of cyst and surgical technique were recorded for the largest cyst.

\subsection{Postoperative management and evaluation}

Postoperatively, all patients were kept prone for several days. Wound healing was classified as healing well, delayed healing, or requiring debridement/suturing. MRI was performed two weeks after surgery. Postoperative radiological evaluation of the sacral canal was performed by a neuroradiologist blind to the patient's intraoperative diagnosis, and was classified as complete cyst resolution, residual cyst, or disappearance of cysts but with effusion into the canal cavity. Postoperative IJOA scores were evaluated three months after surgery.

\subsection{Follow-up}

All patients were followed up until January 2013 by outpatient clinic visits or by telephone questionnaire. The prognosis of each patient was categorized as good or poor.

\subsection{Statistical analysis}

Data analysis was performed using SPSS 17.0 (SPSS, Chicago, IL, USA). Data were compared using the two-samples $t$-test for parametric data and the Mann-Whitney test for non-parametric data. Data are expressed as the mean \pm standard error. $P$-values of less than 0.05 were considered statistically significant.

\section{Results}

\subsection{Demographics}

The 38 patients included in this study were 14 males $(36.8 \%)$ and 24 females (63.2\%). Patients ranged in age from 13 to 70 years, with a mean age of $41.4 \pm 15.57$ years. The mean length of hospital stay was $14.5 \pm 3.84 \mathrm{~d}$.

\subsection{Presentation}

The most common presentation involved symptoms in multiple locations $(47.4 \%, 18 / 38)$, followed by symptoms located at the lower waist $(26.3 \%, 10 / 38)$, legs $(13.2 \%, 5 / 38)$, sacrococcygeal region $(10.5 \%, 4 / 38)$ and buttocks $(2.6 \%$, $1 / 38)$. The most common presenting symptom was pain $(81.6 \%, 31 / 38)$, followed by more than one symptom $(13.2 \%, 5 / 38)$, numbness $(2.6 \%, 1 / 38)$ and a bearing-down feeling $(2.6 \%, 1 / 38)$. The mean period of symptoms before initial presentation was $34.2 \pm 50.28$ months.

\subsection{Nerve function}

Preoperative symptoms included sensory dysfunction in 16 patients, legs weakness in 11 patients and bowel/bladder dysfunction in 10 patients. The mean preoperative IJOA score was $18.8 \pm 1.32$. Preoperative bowel/bladder function was recorded as normal in 28 patients, slight dysfunction in six patients, severe dysfunction in three patients, and incontinent in one patient.

The mean three-month postoperative IJOA score was $19.6 \pm 0.65$, which was significantly different to the preoperative IJOA score $(t=-3.77, P=0.001)$, indicating a significant improvement in neurological function after surgery (Figure 2). The most significant area of improvement in neurological function was sensation $(z=-2.86, P=0.004)$, followed by bowel/bladder function $(z=-2.31, P=0.02)$. Of the 10 patients with preoperative bowel/bladder dysfunction, four still had abnormal bowel/bladder function postoperatively (three had slight dysfunction and one had severe dysfunction).

\subsection{Characteristics of SESMCs}

Eleven patients had a paraneural cyst and 27 had a perineural cyst. The maximum cyst diameter ranged from 1.5 to 9 $\mathrm{cm}$, with a mean maximum diameter of $3.2 \pm 1.54 \mathrm{~cm}$. Seventeen patients had a single cyst, 13 patients had two cysts, and eight patients had three cysts. The mean number of cysts was $1.8 \pm 0.87$. 


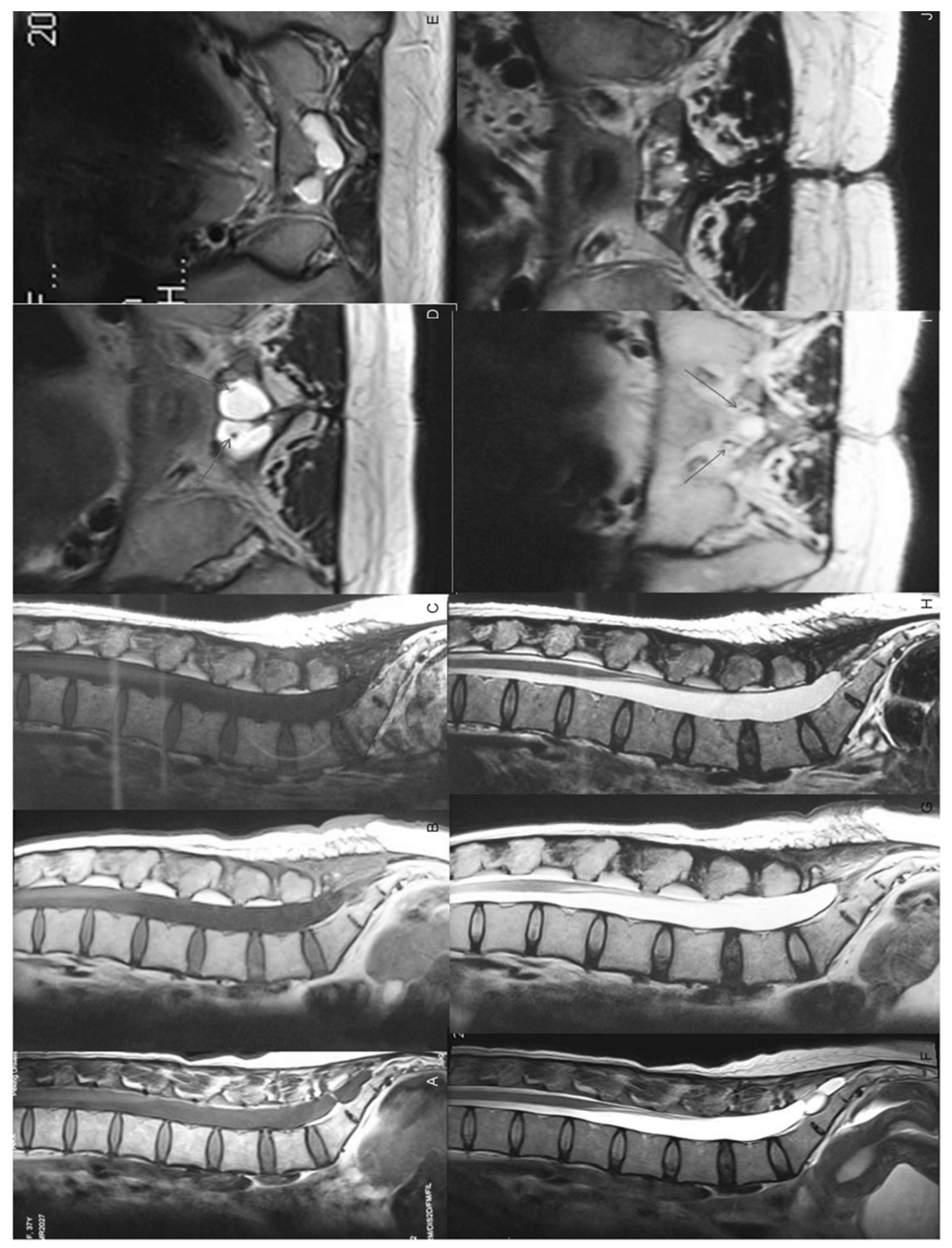

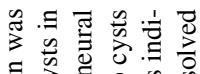

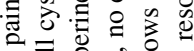

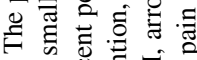

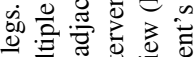

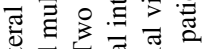

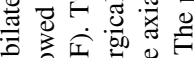

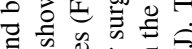

$\vec{x}$ 品 $\overline{0}$

$\sum^{2}$

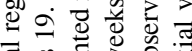

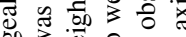

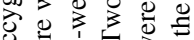

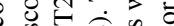

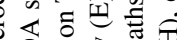

象

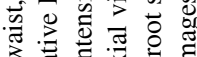

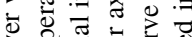

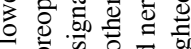

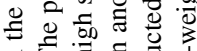

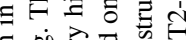

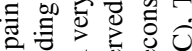

on

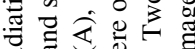

क्ष

등

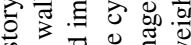

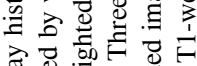

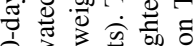

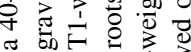

=

3.

递

过

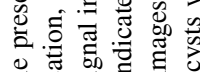

뭉

월 응

在员窘

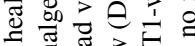

入

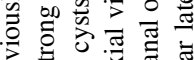

응

可语记

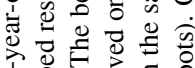

等

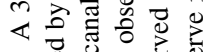

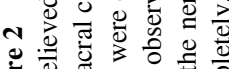

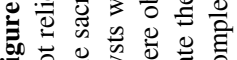

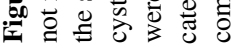




\subsection{Postoperative course}

The mean duration of prone positioning after surgery was $5.5 \pm 3.19 \mathrm{~d}$. Wound healing was classified as well healed in 31 patients, delayed in two patients, and requiring debridement/suturing in five patients. Postoperative radiographic evaluation of the sacral canal was classified as complete cyst resolution in 17 patients, residual small cyst in six patients, and disappearance of cysts but with effusion into the canal cavity in 15 patients.

\subsection{Follow-up and prognosis}

The mean follow-up period was $21.0 \pm 12.54$ months (range 3-43 months). Of the 16 patients with preoperative sensory dysfunction, six still had sensory dysfunction postoperatively. The sensory dysfunction was aggravated in one patient, and was unchanged in the other five patients. Ten patients experienced improved leg muscle strength, and one had remaining leg weakness. The prognosis was classified as good in 37 patients and poor in one patient. The patient with poor prognosis had severe bowel/bladder dysfunction, aggravation of sensory dysfunction, and leg weakness.

\section{Discussion}

Upright walking is a uniquely human behavior, and is the foundation of human civilization. The hydrostatic pressure in the cistern terminalis, the terminal thecal sac, is higher when standing than in other positions. Some individuals may have congenital weakness of the nerve sheath. Similar to the hemodynamic mechanism of aneurysm formation, years of hydrostatic pressure may cause the weak wall to gradually expand outwards into the sacral space. Upright walking therefore underlies the formation of SESMCs. Some authors have attributed cyst formation to the hydrostatic pressure of CSF [1-2,4,7-13].

Enlargement of SESMCs may be caused by a ball-valve effect, congenital abnormalities, connective tissue disorders, and nerve root sheath duplications. The CSF enters cysts with systolic pulsation or with standing and walking, but is unable to exit the cysts through the same portal during diastole or motionless activity. A ball-valve effect of the cyst neck results in a gradual increase in the size of the cyst [14]. This ball-valve effect is the usual mechanism for initiation and development of SESMCs.

During development and enlargement of SESMCs, the adjacent nerve root fibers are stimulated and displaced, causing a variety of symptoms. The most common initial symptom is pain, and multiple anatomic regions are most commonly involved at the time of initial presentation. The specific symptoms and involved regions are related to the anatomy of the sacral canal. Most SESMCs are derived from nerve root sheaths at the L5 to S1 level. Because of the relatively weak dorsal and lateral walls of the sacral canal, the enlarging cysts extend in the dorsal, lateral and downward directions. The sensory root fibers are involved first, followed by nerve roots controlling bowel/bladder function, and lastly the motor nerve roots. Enlargement of SESMCs with SNRFs leads to progression of symptoms. As the mass enlarges, the sensory nerve root fibers are stretched over the periphery of the lesion or are compressed against adjacent nerve roots, causing pain or other sensory disturbance at multiple levels $[2,15]$.

SESMCs with SNRFs often present with multiple smaller cysts. SESMCs with SNRFs (including the paraneurium (Figure $\mathrm{S} 1$ in Supporting Information) and perineurium (Figure S2)) extending around the circumference of the nerve may enlarge to compress the neighboring nerve roots. Patients may begin to feel pain or paresthesiae early, and even very small cysts with SNRFs can therefore be diagnosed early.

SESMCs with SNRFs have been reported to cause sacral pain, sacral fractures, coccygodynia, low back pain, intermittent claudication and lumbosacral radiculopathy. Patients may complain of low back pain, leg pain, and sensory disturbances such as paresthesia or hypoesthesia. Specific radicular pain may result from distortion or compression of nerve roots $[2,15]$. Pain is usually intermittent and is most frequently exacerbated by standing, walking and coughing. Perineural cysts were initially described at the posterior sacral or coccygeal nerve roots [16]. As SESMCs with SNRFs develop over the long term, the motor and bowel/bladder nerve roots fibers may be compressed.

It is generally accepted that asymptomatic SESMCs do not require surgical intervention [1-5,7-19]. When these lesions are symptomatic, the goals of surgical intervention are to relieve nerve stimulation and compression, and stop bone erosion. Even in small SESMCs with SNRFs, nerve root stimulation can cause severe pain that cannot be relieved by lying down or strong analgesic medication and may require urgent surgical intervention. Such symptoms of continuous nerve root stimulation may result from a large increase in the volume of CSF in the cyst over a short period of time, which sharply increases the hydrostatic pressure in the cyst resulting in continuous stimulation of the surrounding nerve root fibers. Opening and partial resection of the cyst relieves compression of the nerve roots and stimulation by hydrostatic pressure. Sensory and bowel/bladder dysfunction are also relieved. It is therefore emphasized that early surgical intervention should be performed for symptomatic SESMCs. In our study, most patients experienced significant improvement in their neurological function after surgery. The most significant area of improvement in neurological function was sensation, followed by bowel/bladder function.

There is still no consensus regarding the appropriate surgical techniques for treating different types of SESMCs. Jung et al. [18] suggested that conservative treatment such as epidural blockade would be useful for patients with peri- 
neural cysts. Patel et al. [20] proposed percutaneous drainage and fibrin glue infusion for the treatment of meningeal cysts of the sacral spine. However, Voyadzis et al. [5] did not recommend percutaneous drainage because of the potential for unexpected nerve root injury. In our experience, fibrin glue infusion may result in pulling of the involved nerve root fibers by the hardened glue. The symptoms of nerve stimulation are not relieved, and may even be aggravated by the fibrin glue. When cysts without involved nerve roots fibers are identified under the microscope, use of fibrin glue to seal the fistula may be considered. Xu et al. [21] reported favorable results after microsurgical cyst fenestration and imbrication.

In our opinion, the redundant cyst wall should be removed, and the communicating fistulae should be oversewn to prevent leakage of CSF from the subarachnoid space and to reconstruct the nerve root sheath. Reconstruction of nerve root sheaths is performed to restore the normal physiological environment of the spinal nerve root fibers. Oversewing of redundant cyst walls aims to strengthen the congenital weakness, and restore the normal outer diameter of the involved nerve root sheath. Two interventions are performed to interrupt the mechanisms causing cyst enlargement. First, to change the unidirectional valve and allow bidirectional flow, the narrow obliterated fistula is enlarged to the size of the outer diameter of the reconstructed nerve root sheath. Second, to overcome the effects of high hydrostatic pressure generated by upright walking, patients are kept prone in bed for several days. Two weeks after surgery, the improvement and recovery of nerve root function are determined by the ongoing alteration of the physiological and biological mechanisms underlying the formation of SESMCs with SNRFs.

It is emphasized that postoperative CSF leakage and cyst recurrence should be avoided by careful microscopic surgical techniques. All communicating fistulas should be identified and transfixed by ligation or oversewn, and a Valsalva maneuver should be performed to ensure that there is no residual leakage. Even with appropriate operative techniques, residual small cysts cannot be completely avoided. Further research is needed to develop surgical techniques and improve treatment. Resection of giant cysts that have developed over a long period of time results in a giant cavity in the sacral canal. As the relatively weak sacrococcygeal muscles and soft tissues cannot effectively fill this cavity, apparent effusion in the canal cavity after surgical intervention is unavoidable. To reduce this effusion in the canal cavity, it is necessary to stay prone in the bed for several days. There is less fluid exudation if the hydrostatic pressure in the cisterna terminalis is kept stable with this positioning. Moderate compression with a $1 \mathrm{~kg}$ clean sandbag results in suitable filling of the canal cavity with relatively weak muscle and soft tissues.
In summary, the goals of surgical treatment for SESMCs with SNRFs are to relieve nerve stimulation and compression, and to stop bone erosion. Most patients experience significant improvement in neurological function after surgery. The most significant improvement in neurological function is sensation, followed by bowel/bladder function.

This work was supported by grants from the Science Foundation for the Excellent Youth Scholars of Ministry of Education of China (200800011035) and the National Natural Science Foundation of China (81200969/H0912).

1 Nabors M W, Pait T G, Byrd E B, et al. Updated assessment and current classification of spinal meningeal cysts. J Neurosurg, 1988, 68: 366-377

2 Tarlov I M. Spinal perineurial and meningeal cysts. J Neurol Neurosurg Psychiatry, 1970, 33: 833-843

3 Cantore G, Bistazzoni S, Esposito V. Sacral Tarlov cyst: surgical treatment by clipping. World Neurosurg, 2013, 79: 381-389

4 Singh P K, Singh V K, Orth D, et al. Tarlov cyst and infertility. J Spinal Cord Med, 2009, 32: 191-197

5 Voyadzis J M, Bhargava P, Henderson F C. Tarlov cysts: a study of 10 cases with review of the literature. J Neurosurg, 2001, 95: 25-32

6 Lingor P, Koch J C, Tönges L, et al. Axonal degeneration as a therapeutic target in the CNS. Cell Tissue Res, 2012, 349: 289-311

7 Acosta F L, Quinones-hinojosa A, Schmidt M H, et al. Diagnosis and management of sacral Tarlov cysts - case report and review of the literature. Neurosurg Focus, 2003, 15: 1-4

8 Park H J, Kim S, Lee S W, et al. Two cases of symptomatic perineural cysts (Tarlov cysts) in one family - a case report. J Korean Neurosurg Soc, 2008, 44: 174-177

9 Ju C, Shin H, Kim S W, et al. Sacral perineural cyst accompanying disc herniation. J Korean Neurosurg Soc, 2009, 45: 185-187

10 Peña E, Llanero M. Painful legs and moving toes syndrome associated with a sacral Tarlov cyst. Parkinson Related Dis, 2011, 17: 645-646

11 Fernandes C, Pinho R, Veloso R, et al. Tarlov cysts: an unusual case of perianal pain. Tech Coloproctol, 2012, 16: 319-320

12 Wang Z Y, Sun J J, Xie J C, et al. Comparative analysis on the diagnosis and treatments of multi-segment intramedullary spinal cord tumors between the different age groups. Neurosurg Rev, 2012, 35 : 85-93

13 Orahilly R, Muller F, Meyer D B. The human vertebral column at the end of the embryonic period proper. The sacrococcygeal region. $\mathrm{J}$ Anat, 1990, 168: 95-111

14 Landers J, Seex K. Sacral perineural cysts: imaging and treatment options. Br J Neurosurg, 2002, 16: 182-185

15 Freidenstein J, Aldrete J A, Ness T. Minimally invasive interventional therapy for Tarlov cysts causing symptoms of interstitial cystitis. Pain Phys, 2012, 15: 141-146

16 Duja C M, Berna C, Kremer S, et al. Confusion after spine injury: cerebral fat embolism after traumatic rupture of a Tarlov cyst: case report. BMC Emerg Med, 2010, 10: 18

17 Slipman C W, Bhat A L, Bhagia S M, et al. Abdominal pain secondary to a sacral perineural cyst. Spine J, 2003, 3: 317-320

18 Jung K T, Lee H Y, Lim K J. Clinical experience of symptomatic sacral perineural cyst. Korean J Pain, 2012, 25: 191-194

19 Tanaka M, Nakahara S, Ito Y, et al. Surgical results of sacral perineural (Tarlov) Cysts. Acta Med Okayama, 2006, 60: 65-70

20 Patel M R, Louie W, Rachlin J. Percutaneous fibrin glue therapy of meningeal cysts of the sacral spine. AJR, 1997, 168: 367-370

21 Xu J Q, Sun Y D, Huang X, et al. Management of symptomatic sacral perineural cysts. PLoS ONE, 2012, 7: e39958

Open Access This article is distributed under the terms of the Creative Commons Attribution License which permits any use, distribution, and reproduction in any medium, provided the original author(s) and source are credited. 


\section{Supporting Information}

Figure S1 One case with paraneural cyst.

Figure S2 One case with perineural cyst.

The supporting information is available online at life.scichina.com and www.springerlink.com. The supporting materials are published as submitted, without typesetting or editing. The responsibility for scientific accuracy and content remains entirely with the authors. 